\title{
Facial Plexiform Neurofibromatosis
} Type I

Bapan Devnath, ${ }^{1}$ Debraj Dey, ${ }^{2}$ Avinava Ghosh, ${ }^{3}$

\begin{abstract}
Introduction
Plexiform neurofibroma is a benign tumor of peripheral nerves arising from a proliferation of all neural elements. Clinically, it presents as a subcutaneous mass which feels like a "bag of worms".

Case Report

A 23-years-old male with a swelling over left side of the face large enough to involve whole one side of the face. There was tenderness and the swelling had a very peculiar consistency, soft in most of the areas with few firm nodular areas.

Operative procedure: surgery was done in two stages. Initially subtotal excision was done and 3 months later re-explored to excise the recurrent disease. Complete excision was not possible.

Discussion

Plexiform neurofibroma (PNF) occurs due to overgrowth of neural tissue in the subcutaneous region. Surgical management remains the mainstay of treatment but functional disturbances are almost inevitable while resecting tumors involving the head and neck region. Resection and de bulking of invasive PNF is however associated with a high rate of recurrence. One of the limiting factors is vascularity of these lesions and their abnormal propensity to bleed.

Conclusion

Although benign, plexiform neurofibromas can cause pain, disfigurement and functional changes and more importantly, may turn malignant. Surgery should be attempted considering psychological benefits.

$\underline{\text { Keywords }}$

Neurofibroma, Plexiform; Facial Asymmetry; Male
\end{abstract}

ABSTRACT

$\mathrm{N}$ eurofibromatosis (NF) type 1 (von Recklinghausen NF) is a genetic disorder that occurs in 1 of 4000 births. It is inherited in an autosomal dominant pattern with variable penetrance; however, as many as $50 \%$ of cases may result from spontaneous mutation. The disease results from a defect in a tumor suppressor gene on chromosome 17, which leaves affected individuals at risk for developing a variety of benign and malignant tumors. ${ }^{1}$ Plexiform neurofibroma is a benign tumor of peripheral nerves (WHO grade I) arising from a proliferation of all neural elements, pathognomonic of NF1. It involves single or multiple nerve fascicles that arises from major nerve branches.

These are among the most common and debilitating types of neurofibromatosis type 1 (NF1). Clinically, it presents as a subcutaneous mass which feels like a "bag of worms". ${ }^{2}$ Most of the time, it is a superficial cutaneous and subcutaneous lesion, but it can occur almost anywhere in the body. It is found in approximately $30 \%$ of patients with neurofibromatosis type 1 and the massive form results in functional disability and disfigurement. Such tumors are generally present at birth, and often progress slowly during early childhood. There is a risk of malignant transformation into a malignant peripheral nerve sheath tumor (MPNST) in 5-10\% of cases. ${ }^{3,4}$

There is no established means of medical treatment, but research into the molecular pathogenesis of NF1, as well as advances in tumor therapy in general, are opening the way towards clinical trials for plexiform neurofibroma. ${ }^{5}$ Most cases require repeat surgery as

1 - Department of ENT, Silchar Medical College, Assam

2 - Department of ENT, Tezpur Medical College \& Hospital, Assam

3 - Department of ENT, Jain ENT Hospital, Jaipur,

Rajasthan

Corresponding author:

Dr Bapan Devnath

email: devnathbapan@gmail.com 


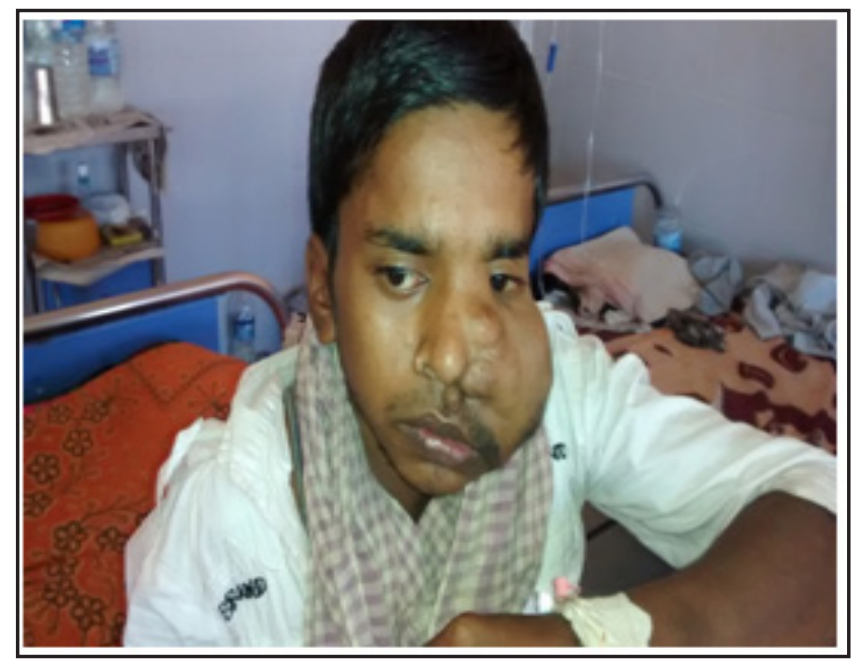

Fig.1. Pre-op clinical photograph of facial plexiform NF

complete excision is generally not possible due to the infiltrating nature of these tumors. Such neurofibromas infiltrate multiple tissue planes and are thus much more difficult or impossible to resect completely. ${ }^{6}$

\section{Case Report}

A 23-year-old male presented with a chief complaint of a swelling on the left side of the face since five years. After an asymptomatic period of 4 years the patient had started to experience mild, intermittent dull aching pain in the swelling for one year. There was no history of any regression in size of swelling or any discharge or trauma in the left side.

The patient had never noticed similar swellings elsewhere in the body. The past medical, surgical, and dental histories were unremarkable. Family history did not reveal any similar complaint in immediate or distant relatives. General physical examination showed a moderately built and well nourished 23-year-old male with steady gait, and satisfactory vital signs. There was no palpable lymph node in the neck or any site of the body.

There were no sign of icterus, clubbing or anemia. On local examination a diffuse swelling on the left side of the face extending up to the lateral wall of the left nostril up to root of the nose above and upper lip below. (Fig. 1) Laterally it extended up to $2 \mathrm{~cm}$ medial to the

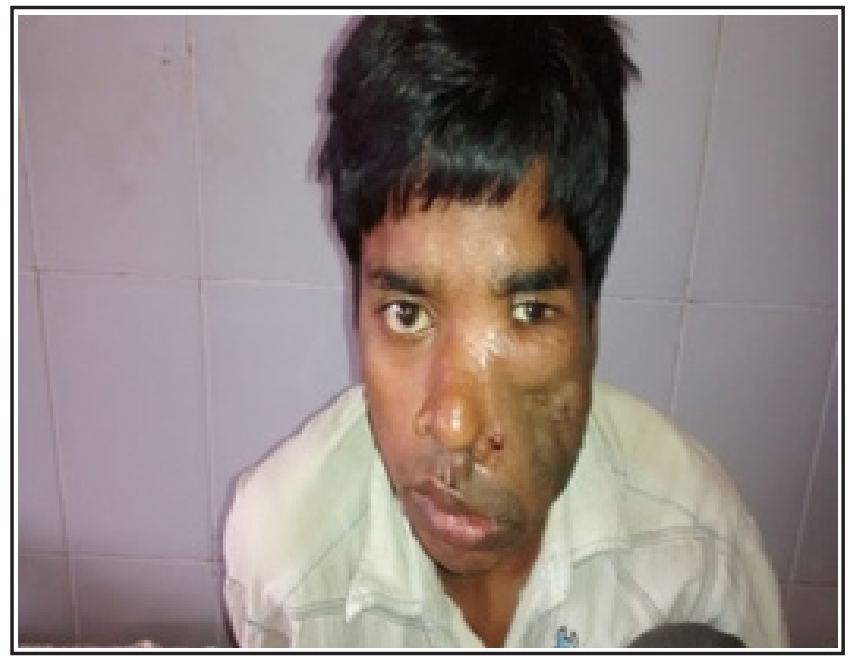

Fig. 2. Post-op photograph after 3 months with recurrence

pre-auricular region and lateral border of mandible, it also involved the left lower eye lid but vision on that side was normal. The swelling measured approx 10x10 $\mathrm{cm}$ in size and had indistinct borders. He had few small (around 5-8mm) neurofibromatous lesions over other side of the face and forehead. No other lesions were seen in any other site of the body.

On palpation, there was mild tenderness and the swelling had a very peculiar consistency, soft in most of the areas with few firm nodular areas, similar to that described in literature as a 'bag of worms'. No pitting was noticed. On auscultation no bruit could be detected. Intra-oral examination showed no focus of pathology, and the swelling had not extended intra orally.

Based on the history and clinical presentation, a working diagnosis of Facial Neurofibroma with neurofibromatosis type I (plexiform neurofibroma) was given. For the facial swelling however, the differential diagnosis included hemangioma, lymphangioma and arterio-venous malformation.

The patient was subjected to radiographic investigations. The panoramic and postero-anterior skull views did not reveal any evidence of pathology. Computed tomography showed an irregular hypodense enhancing soft tissue mass lesion confined to the subcutaneous tissue of the left cheek. No bony involvement was evident. Complete hemogram showed values that were within the normal range. 


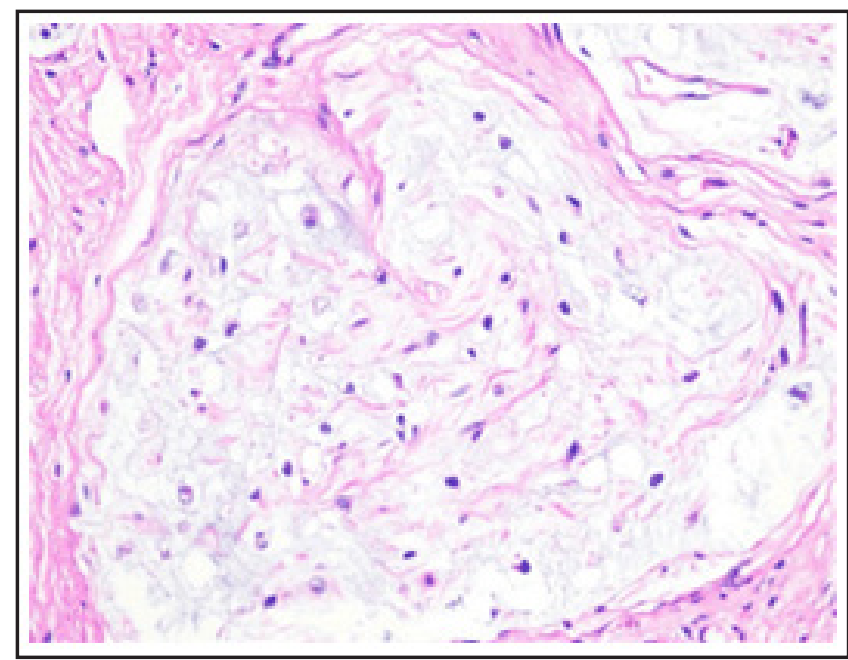

Fig. 3. Histopathological section of Plexiform neurofibroma; showing neural elements and fibroblasts

Lateral rhinotomy incision was given and facial skin flap was raised under general anesthesia. Operative findings revealed grossly thickened subcutaneous nerves and neo-vessels in the tumor mass, which was infiltrating deeply, and no clear tissue planes were identifiable. The neo-vessels were very friable and bled profusely during dissection. Complete excision was not possible; hence, subtotal excision and re-draping of skin flap was carried out.

The post-operative period was uneventful. However, on review after 3 months, there was partial re-growth of the swelling. (Fig. 2) The histopathological sections showed a lesion composed of bundles of nerve fibers arranged in a concentric manner with areas of myxoid changes. Schwann cells and fibroblasts were also seen. A histopathological diagnosis of Plexiform Neurofibroma was established. (Fig. 3)

In the second setting a curvilinear incision was given around $1.5 \mathrm{~cm}$ below the left lower eye lid, then the skin flap was raised and the remaining resectable tumor mass were resected. Closure of operative wound done in layers. This whole procedure was done in local anesthesia under sedation. Few days in the immediate post operative period he had lower lid edema which got subsided completely after a week. (Fig. 4)

The case was then submitted to a physician and to rule out other features of neurofibromatosis-I and also

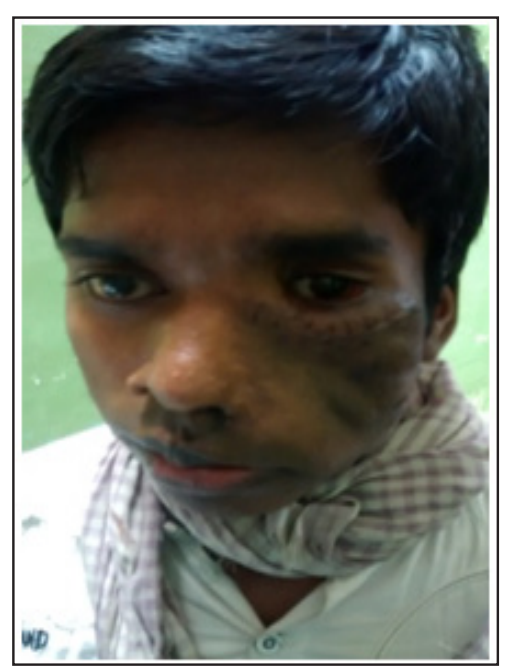

Fig. 4. Post-op. photograph 7 days after final operation

to rule out the possibility of any systemic involvement.

\section{Discussion}

Characteristics of NF1 is occurrence of peripheral nerve sheath tumors, Neurofibromas which are most common cause of disfigurement in NF1.The term plexiform NF used to describe a network like growth of tumor involving multiple fascicles of nerve leading to diffuse mass of thickened nerve fibers surrounded by proteinaceous matrix. ${ }^{7} \mathrm{PNF}$ is a rare type of generalized $\mathrm{NF}$, which occurs due to overgrowth of neural tissue in the subcutaneous tissue. The diffuse and soft nature of PNF is often compared with "a bag of worms."

Localized NF1 usually present along a peripheral nerve and increases at late childhood and early adolescence, with a well defined margin mostly over skin also in stomach, kidney, bowels, bladder. ${ }^{8}$

The lesions can occur anywhere along a nerve, and may appear on the face, orbit and globe and frequently involve the cranial and upper cervical nerves. The condition can be quite disfiguring, as observed in the cases being presented, and hemifacial hypertrophy can occur. Complications include bleeding from trauma, neurological deficits and psychological disturbance because of abnormal anatomy. ${ }^{9}$

Histologically, these are peripheral nerve sheath 
tumors containing all elements of the peripheral nerve, and are characterized by an increase in endoneural matrix with separation of nerve fascicles and proliferation of Schwann cells. PNF can turn malignant in $4-5 \%$ of cases. ${ }^{10}$

Resection and debulking of invasive PNF is however associated with a high rate of recurrence. In one pediatric series, complete resections developed recurrence in $20 \%$ and incomplete resections had a recurrence in up to $45 \%$ of the cases. One of the limiting factors is vascularity of these lesions and their abnormal propensity to bleed. These tumors bleed profusely during surgery because of the friable nature of the neo-vessels. Adequate blood should be arranged before taking up these cases for resection. ${ }^{7}$

Needle et al. analyzed the largest series of surgically managed PNFs and demonstrated that 54\% recurred within a 10-year period, with the greatest risk of recurrence found in lesions involving the head and neck region. We encountered recurrence in both the cases being presented here. ${ }^{6}$

Wise et al. treated 39 patients of PNF with surgeries, they found $100 \%$ recurrence rate in massive PNFs and $25 \%$ recurrence in small tumors. ${ }^{11}$

Waggnor et al. in their retrospective study of 69 patients of PNFs, 44\% were detected by age 5 and 2 of them found to be MPNS tumors and there were no specific clinical features in NF1 patients. ${ }^{12}$

Surgical management remains the mainstay of treatment for these tumors, but functional disturbances are almost inevitable while resecting tumors involving the head and neck region. No chemotherapeutic agent has yet been identified that reduces the size of these tumors.

\section{Conclusion}

Although characteristically benign, plexiform neurofibromas can cause pain, disfigurement and functional changes and more importantly, may turn malignant. The one salient feature found in all cases of NF-I is its progressive nature. Thus the general trend in each case is towards a worsening of the disease.

Extensive surgical resections in the head and neck region are risky due to the infiltrating nature of these tumors, which can lead to functional disability and a high rate of re growth. Hence, surgery should be undertaken only after giving due consideration to the possible psychological benefits by making these patients socially more acceptable in the society. Periodic clinical examination and MRI evaluation are required for about 2 years for timely detection and repeat surgery to achieve further correction. The role of a cognizant clinician lies in early diagnosis of the condition and alerting the patient about its future complications.

\section{References}

1. Greinwald J, Derkay CS, Schecter GL. Management of massive head and neck neurofibromas in children. Am J Otolaryngol. 1996; 17:136-42

2. Jeffrey B Wise, Jonathan E Cryer, Jean B. Belasco. Management of head and neck plexiform neurofibromas in pediatric patients with neurofibromatosis type 1 . Arch Otolaryngol Head Neck Surg. 2005;131(8):712-8

3. Abbas O, Bhawan J. Cutaneous plexiform lesions. J. Cutan. Pathol. 2010; 37(6): 613-23

4. Huson SM, Hughes RA. The Neurofibromatosis: A Pathogenetic and Clinical Overview. London: Chapman and Hall Medical; 1994

5. Friedrich RE, Schmelzle R, Hartmann M, Fünsterer C, Mautner VF. Resection of small plexiform neurofibromas in neurofibromatosis type 1 children. World J Surg Oncol. 2005; 3:3-6

6. Needle MN, Cnaan A, Dattilo J, Chatten J, Phillips PC, Shochat S, et al. Prognostic signs in the surgical management of plexiform neurofibroma: The Children's Hospital of Philadelphia experience, 1974-1994. J Pediatr. 1997; 131:67882

7. Misra SR, Maragathavalli G, Baskaran P, Rastogi V. Facial plexiform neurofibroma in a 13-year-old girl with neurofibromatosis-1. J Indian Acad Oral Med Radiol. 2012; 24:243-9

8. Patil K, Mahima VG, Shetty SK, Lahari K. Facial plexiform neurofibroma in a child with neurofibromatosis type I: A case report. J Indian Soc Pedod Prev Dent. 2007; 25:30-5

9. Dogra Bharat B, Rana Karan. Facial plexiform neurofibromatosis; a surgical challenge. Indian Dermatol Online J. 2013; 4(3): 195-8

10. Wenig BM. Philadelphia, Pa: WB Saunders Co; 1993. Atlas of Head and Neck Pathology; pp. 156-9

11. Wise JB, Cryer JE, Belasco JB, Jacobs I, Elden L. Management of head and neck plexiform neurofibromas in pediatric patients 
with neurofibromatosis type 1 . Arch Otolaryngol Head Neck Surg. 2005; 131:712-8
12. Waggoner, Towbin J, Gottesman G, Gutmann DH. Clinic based study of plexiform neurofibromas in NF1. Am J Med Genet. 2000; 92(2):132-5. 